\title{
A Novel Hybrid Block INF Diagonalization (HBID) Precoding Technique for Capacity Improvement in MU-MIMO-OFDM System
}

\author{
R. Santhakumar, N. Amutha Prabha
}

\begin{abstract}
In wireless communication system, the emerging technology is MU-Multiple Input Multiple Output-Orthogonal Frequency Division Multiplexing (MU-MIMO-OFDM). For minimizing Peak-to-Average Power Ratio (PAPR) in the uplink transmission scheme, OFDM is exploited in MIMO system and compared with Single Carrier-Frequency Domain Processing (SC-FDP). Further reducing the PAPR value, a new method is proposed Hybrid Block Inf Diagonalization (HBID) technique for an uplink OFDM system and calculated the less Bit Error Rate (BER). In this paper, the HBID precoding technique reduces PAPR with such amount and unwanted amplitude of the signal is to be cut down for a specific threshold value as 1.4 for best performance so that the PAPR value is reduced in considerable rate of OFDM then compared to SC-FDP system. Also, we compared the various precoding techniques with HBID method in MIMO-OFDM systems with various parameters such as BER, PAPR, Number of users, Sum rate. Finally, the proposed method reduces PAPR with $47 \%$ and BER with 50\%, number of users with 49\% and sum rate as 57\%. HBID based precoding method shows its excellence with the lowest PAPR value and thereby enhance the presentation of the OFDM system
\end{abstract}

Keywords : About four key words or phrases in alphabetical order, separated by commas.

\section{INTRODUCTION}

In modern scenario, communication device plays a major role in every one's life. The communication device is a hardware device such as Smart Phones, Bluetooth devices, Modem, Wi-Fi devices and etc. which is used to transmit or receive information through the wire as a medium that is wired communication or through the air as a medium known as wireless communication. The major limitations of wired communications are: the equipment is not portable, it requires more space, people are restricted to some particular places, the cables or wires can be easily damaged, the power consumption is more and etc. To overcome these limitations, wireless communication is preferred and it has some advantages like easy installation, flexibility, low power consumption, increased efficiency and so on. Same way, it has some demerits like interference, unsecure transmission of signal from transmitter to receiver and so on.

In wireless communication, many modulation techniques are used such as BPSK, QPSK, QAM and so on but one of the

Revised Manuscript Received on November 08, 2019.

R.Santhakumar, School of Electrical Engineering, Vellore Institute of Technology, Vellore, Tamil Nadu, India.

*N. Amutha Prabha, School of Electrical Engineering, Vellore Institute of Technolgoy, Vellore, Tamil Nadu, India important modulation schemes is Orthogonal Frequency Division Multiplexing (OFDM). It uses multiple carriers for transmitting a large amount of data. There are many advantages present in the OFDM systems. They are high tolerant to frequency selective fading channel, high spectrum efficacy, high bitrate for transmission and etc. The major limitations are: high PAPR due to it requires large number of $\mathrm{RF}$ power amplifiers to reduce an undesired magnitude, Out of Band $(\mathrm{OoB})$ radiation, in-band distortion and it is highly sensitive to CFO. In addition to OFDM system, in wireless communication, instead of using a single antenna at the transmitter and receiver multiple antennas are used which is known as MIMO system. In this technique, multiple antennas are connected at the transmitter and receiver so multiple signals are transmitted via a different number of antennas at the transmitter by using the same frequency and separated space thereby increase the quality of transmission of data, increase the operational speed and provide security to the network [1]. Because of these merits,these MIMO techniques have been mostly used in recent communication systems during last decades [2], [3]. The demand for higher area coverage, reliability, diversified usage the MIMO system has to be improved to satisfy this requirement which has been proposed in [4].

The transmitter and receiver are vulnerable to high complexity by implementating Fast Fourier Transform (FFT) and Inverse Fast Fourier Transform (IFFT) in every antenna of MIMO system. Also, MIMO system can minimize the flat fading effects and noise in the uncorrelated receiver through orthogonality and increasing the dimensions of matrices because the antenna number is large which is proposed in [5]. For downlink process, it is necessary to add RF chains fora large antenna system that is MIMO system for transmitting the signal with low distortion. This addition of RF chain causes a major challenge in OFDM system when the carriers are increased [6].

\section{RELATED WORKS}

To cope the problem arises from OFDM system, MIMO technology is used which improves the spectral and power efficiency in a considerable range [7], [8]. The large-scale (LS) antenna structure used in wideband frequency selective channel causes some limitations such as higher complexity, high power consumption, high rate of RF chains. To overcome these limitations Single Carrier Spatial Modulation (SC-SM) transmission Technique is proposed by [9]. 


\section{A Novel Hybrid Block INF Diagonalization (HBID) Precoding Technique for Capacity Improvement in MU-MIMO-OFDM System}

The transmit antenna process in SM system which has been handled by SC-SM architecture. This gives the benefits of the low complexity, low-cost RF transmitter. The downlink process performance of massive Multiuser (MU) MIMO system based on OFDM system is evaluated by implementing the base station with 1-bit digital to analog converters which has been proposed in [10]. By using Bussgang's theorem along with some linear precoding technique such as Zero forcing and maximal ratio transmission, the performance of the system can be described.

In 5th generation technology, the spectral efficiency and energy efficiency can be obtained for MIMO which operates in millimeter wave frequencies. Two single carrier modulation schemes are to be considered. They are: usual modulation technique along with linear equalization and single carrier modulation with cyclic prefix, equalization based on frequency domain and processing based on FFT. These are compared with a conventional MIMO-OFDM transceiver architecture which has been proposed in [11]. Linear equalization can be used in traditional modulation whereas frequency domain modulation can be used in single carrier modulation and analyze and compare the two techniques. For efficient implementation, pilot contamination in time division duplex mode is taken into account. Uplink and downlink channels can be calculated in a base station in massive MIMO using the uplink pilot training in conjunction of time division duplex with channel reciprocity property. During uplink training, the non-orthogonal pilot signals are used which creates the contamination in the channel estimation. This leads to the interference between adjacent cells. So, this pilot contamination and elimination method have been proposed in [12] in addition with zero-forcing precoding in massive MIMO systems. The scheme proposed the base station which is allocated to the orthogonal variable spreading factor (OVSF) and Zadoff-Chu (ZC) signals are used for uplink training. Repeatedly add the ZC sequence at each base station and OVSF generates the orthogonality across the adjacent cell. So, this method provides the uncontaminated channel estimation and reduces interference without increasing training overhead.

For downlink process, precoding technique is applied at the base station in multiple antenna systems with multi-cell which is presented in [13]. The base station is precoded based on the Channel State Information (CSI) which has been obtained by the time division duplex operation. The non-orthogonal training signal is used for uplink training. This affects the precoding matrices in one cell by other cell users. A precoding method relied on novel multi user MMSE [13] to mitigate the pilot contamination issue. A single carrier transmission scheme which has presented in [14] for frequency selective multi-user (MU) multiple input single output (MISO) and the base station which has M number of antennas and $\mathrm{K}$ number of single antenna users. The system has very low complexity and achieves optimal sum-rate performance at low power transmission to noise power ratio. This proposed system provides the receiver without equalization process, it does not need any multi-user resource allocation and it does not depend upon the channel power delay profile.

Least Square (LS), MMSE are some of the technique used to evaluate the MIMO channel which has been presented in [15]. Based on the proposed method in [15] the performance of the Training Based Channel Estimation (TBCE) can be enhanced by increasing the number of transmitter and receiver antenna increased. For all types of modulation schemes, the performance of MMSE and LS estimations are same at small SNR value but once the SNR value is to be increased then the performance gap also goes on increasing. Recently, in MIMO system the time domain equalization is very complex to implement. So to perform the equalization in receiver side single carrier frequency domain equalization is proposed in [16] which use the MMSE criterion. Paper [17] analyze both the systems such as OFDM and SC-FDP in the presence of non-linear power amplifier and derive the sum rate of both zero forcing and matched filter precoding scheme. Paper [20] shows the block diagonalization technique for MIMO system and the PAPR reduction performance. Paper [21] described hybrid precoder for MU schemes and the derivation for uplink and downlink.

\section{SYSTEM MODEL}

MU-MIMO framework is appeared in fig 1 . The framework comprises of $\mathrm{N}_{\mathrm{BS}}$ antenna at base station and $\mathrm{M}_{\mathrm{BS}}$ $\mathrm{RF}$ chain at RF module, each of is accepted to plan $\mathrm{K}$ versatile station. Each MS is ready with $\mathrm{N}_{\mathrm{MS}}$ antennas and $\mathrm{M}_{\mathrm{MS}} \mathrm{RF}$ chains to support $\mathrm{N}$ information streams, which implies add up to $\mathrm{K} \times \mathrm{N}_{\mathrm{S}}$ information streams are dealt by the $\mathrm{BS}$. To ensure the adequacy of the communication carried by the constrained number of RF chains, the number of the transmitted steams is obliged by $\mathrm{K} \mathrm{N}_{\mathrm{S}}=\mathrm{M}_{\mathrm{BS}}=\mathrm{N}_{\mathrm{BS}}$ f or the BS and $\mathrm{N}_{\mathrm{S}} \leq \mathrm{M}_{\mathrm{MS}} \leq \mathrm{N}_{\mathrm{BS}}$ for each MS.

At the BS, the transmitted data are accepted to be prepared by a baseband precoder $\mathrm{B}$ of measurement $\mathrm{M}$ and after that by an RF precoder $\mathrm{F}$ of measurement. Outstandingly, the baseband precoder B empowers both sufficiency and stage modification, whereas as it were stage changes (phase-only control) can be realized by F since it is actualized by utilizing analog stage shifters. Each section of $\mathrm{F}$ is normalized to fulfill $|F(i, j)|=\frac{1}{\sqrt{N_{B S}}}$, where $|\mathrm{F}(\mathrm{i}, \mathrm{j})|$ represents the amplitude of the $(i, j)^{\text {th }}$ component of $F$. Additionally, to encounter the total transmit power limitation, $\mathrm{B}$ is standardized to fulfill $\|\mathrm{FB}\|^{2}$, where $\|\cdot\|$ the Frobenius norm.

The received signal of the $\mathrm{k}^{\text {th }} \mathrm{MS}$

$$
y_{k}=F_{k} F B_{s}+n_{k}, k=0,1,2, \ldots, K
$$

where $s \in C^{K N_{S} \times 1}$ is the signal vector for a total of $\mathrm{K}$ MSs, each of which forms a NS $\times 1$ signal vector $S_{K}$. Namely $s=\left[S_{1}^{T}, S_{2}^{T}, S_{3}^{T}, \ldots, S_{K}^{T}\right]^{2}$, where $(.)^{2}$ represents transpose. And the signal vector satisfies the demands of $E\left[S S^{H}\right]=$ $\frac{\rho}{K N_{S}} I_{K N_{S}}$, where $(.)^{\mathrm{H}}$ denotes conjugate transpose, $\mathrm{E}[$. signifies expectation, $\rho$ is the average transmit power and $I_{K N_{S}}$ is the $K N_{S} \times K N_{S}$ identity matrix. $F_{k} \in C^{N_{M S} \times N_{B S}}$ is the channel matrix for the $\mathrm{k}^{\text {th }} \mathrm{MS}$, and $N_{k}$ is the $\mathrm{N}_{\mathrm{MS}} \times 1$ vector of i.i.d. $\varepsilon N\left(0, \sigma^{2}\right)$ additive complex Gaussian noise. Received signal at the $\mathrm{k}^{\text {th }}$ MS after merging is given by 
$\bar{y}_{k}=T_{k}^{H} Y_{k}^{H} F_{k} F B s+T_{k}^{H} Y_{k}^{H} n_{k}, k=1,2, \ldots, K$,

Where $Y_{k}$ is the $N_{M S} \times M_{M S}$ RF joining matrix and $M_{k}$ is the $M_{M S} \times N_{S}$ baseband joining matrix for the $\mathrm{k}^{\text {th }} \mathrm{MS}$. Since $W_{k}$ is also realized by the analog phase shifters, all fundamentals of $Y_{k}$ should have the continuous amplitude such that $\left|Y_{k}^{(i, j)}\right|=\frac{1}{\sqrt{N_{B S}}}$. An Eq-baseband channel for each MS as

$$
\widetilde{F}_{K}=Y_{k}^{H} F_{k} F, k=1,2, \ldots, K,
$$

and the entire corresponding MU baseband channel can be represented as

$F_{e q}=\left[\begin{array}{c}\tilde{F}_{1} \\ \tilde{F}_{2} \\ \vdots \\ \tilde{F}_{k}\end{array}\right]=\left[\begin{array}{cccc}Y_{1}^{H} & 0 & \ldots & 0 \\ 0 & Y_{2}^{H} & \ldots & 0 \\ \vdots & \vdots & \ddots & \vdots \\ 0 & 0 & \ldots & Y_{k}^{H}\end{array}\right]\left[\begin{array}{c}F_{1} \\ F_{2} \\ \vdots \\ F_{k}\end{array}\right] F$

Then the processed received signal at the k-th MS can also be represented as

$$
\tilde{y}_{k}=T_{k}^{H} \tilde{F}_{k} C_{k} s_{k}+\sum_{i=1, i \neq k}^{K} T_{k}^{H} \tilde{F}_{k} C_{i} s_{i}
$$

$+T_{k}^{H} Y_{k}^{H} n_{k}, k=1,2, \ldots, K$

Where $\mathrm{C}_{\mathrm{k}}$ is the $\left((k-1) N_{s}+1\right)^{t h}$ to the $\left(k N_{s}\right)^{t h}$ columns of $\mathrm{C}$, corresponding to the baseband precoding for $\mathrm{S}_{\mathrm{k}}$. When the Gaussian symbols are used by the BS, the sum spectral efficiency achieved will be

$Q=\sum_{k=1}^{K} \log _{2}\left(\left|O_{N_{S}}+\frac{P}{K N_{S}} Q_{i}^{-1} T_{k}^{H} \tilde{F}_{k} C_{k} C_{k}^{H} \tilde{F}_{k}^{H} T_{k}\right|\right)$

where $Q_{i}=\frac{P}{k N_{s}} \sum_{i=1, i \neq k}^{K} T_{k}^{H} \tilde{F}_{k} C_{k} C_{k}^{H} \tilde{F}_{k}^{H} T_{k}+\sigma^{2} T_{k}^{H} Y_{k}^{H} T_{k} Y_{k}$ is the covariance matrix of both interference and noise.

For the most part, joint optimization on the RF and baseband precoders and combiners ought to be an fundamental strategy to plan the preparing plot that accomplishes ideal whole ghastly effectiveness $\mathrm{R}$. Be that as it may, as expressed in [15], finding worldwide optima for comparative compelled joint optimization issues (maximizing $\mathrm{R}$ whereas constant-amplitude limitations forced to the RF analog precoder and combiners) is regularly found to be unmanageable. Indeed, within the conventional MU-MIMO frameworks without crossover handling structure, it moreover needs colossal endeavors to discover a neighborhood ideal of entirety rate by substituting optimization [19]. For a few as of late outlined crossover handling schemes [12], [17]- [18] within the writing, isolated $\mathrm{RF}$ and baseband preparing plans are examined to get fulfilling execution without including a horde of iterative strategies. Subsequently, we select to isolate the RF and baseband space plans in this paper.

\section{PROPOSED METHOD}

The proposed method is shown in fig1. It consists of $\mathrm{H}$-infinity precoding, $\mathrm{Rf}$ coder and CEM technique. The data streams are mapped using mapping concept and the outcome from mapping is given to $\mathrm{H}$-infinity precoder. The preliminary objective of precoder is to generate precoding matrix afterwards is given to RF precoder which done the block diagonalization operation and afterward CEM operation is performed. The combination of $\mathrm{H}$-infinity precoding and RF precoder is called HBID (Hybrid Block Inf Diagonalization).

Fig.1: Proposed Transmitter Block Diagram
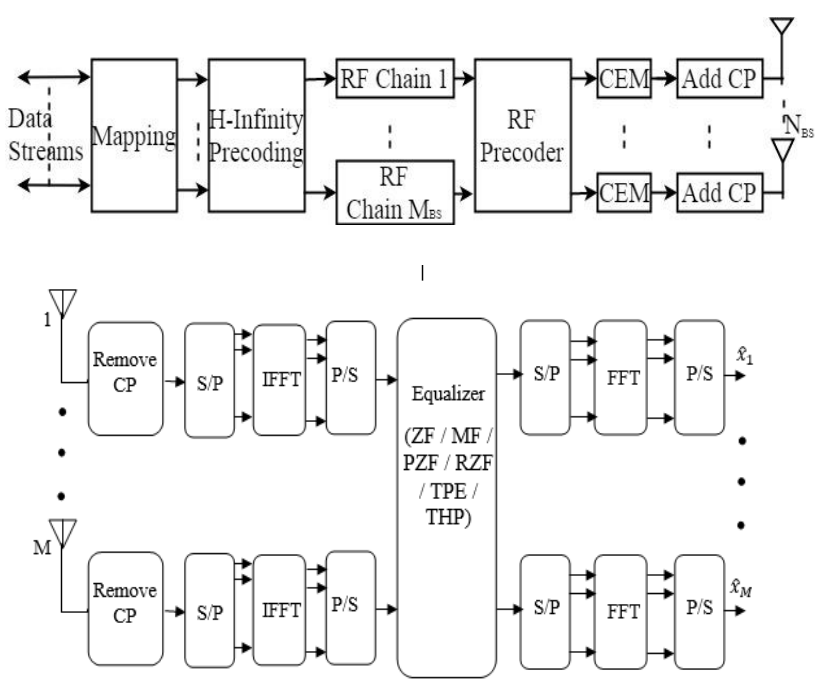

Fig. 2: Receiver Block Diagram $\boldsymbol{x}_{\boldsymbol{k}}$

\section{A. H-infinity precoding:}

The center thought of the $\mathrm{H}$-inf is to discover a precoding matrices B, so that the proportion between the precoding appraise mistake within the $\mathrm{j}^{\text {th }}$ cell and the complete interference/noise in all other cells is less than a endorsed bound. The objective work is communicated as

$$
\tilde{F}_{q j} \hat{B}_{j} G_{j+} x_{j} \frac{\left\|\tilde{F}_{j j} \hat{B}_{j} G_{j}\right\|_{Y}^{2}}{\left\|\sum_{q \neq j}^{Q} \tilde{F}_{q j} \hat{B}_{j} G_{j+} x_{j}\right\|^{2}}<s
$$

Where $\hat{B}_{j}$ is the guesstimate of $B_{j}$ and (10) is equal to, $\mathrm{L}$ (8)

To get a result by using Krein Space, (11) is casted into an indefinite quadratic for

$\left(\left[\begin{array}{c}Z_{j} \\ F_{j j} \hat{B}_{j} G_{j}\end{array}\right]-\left[\begin{array}{c}\tilde{F}_{j j} \hat{B}_{j} \\ I_{K}\end{array}\right] G_{j}\right)^{F}\left[\begin{array}{cc}O_{K} & 0 \\ 0 & -s^{-1} Y\end{array}\right] \times\left(\left[\begin{array}{c}Z_{j} \\ \tilde{F}_{j j} \hat{B}_{j} G_{j}\end{array}\right]-\right.$

\section{FjjBjOKGj>0}

The Krein Space system is given by

$$
\left[\begin{array}{c}
U_{j} \\
G_{j}^{\prime}
\end{array}\right]=\left[\begin{array}{c}
\widetilde{F}_{j j} \\
O_{K}
\end{array}\right] G_{j}+\left[\begin{array}{c}
x_{j}^{\prime} \\
\widetilde{G}_{j}
\end{array}\right]
$$

Where $G_{j}^{\prime}=\tilde{F}_{j j} \hat{B}_{j} G_{j}, \widetilde{H}_{j j}=\tilde{F}_{j j} \hat{B}_{j}, \quad \tilde{G}_{j}=G_{j}^{\prime} G_{j}$,

$x_{j}^{\prime}=\sum_{q \neq j}^{Q} \tilde{F}_{q j} G_{j} G_{j} x_{j}$. Multiplying $\tilde{F}_{j j}^{-1}$ both sides of the upper part of (13), it can be rewritten as

$$
\left[\begin{array}{c}
\varphi_{j} \\
G_{j}^{\prime}
\end{array}\right]=\left[\begin{array}{c}
O_{K} \\
O_{K}
\end{array}\right] G_{j}+\left[\begin{array}{c}
\widetilde{F}_{j j}^{-1} x^{\prime} \\
\tilde{G}_{j}
\end{array}\right]
$$

Where, $\varphi_{j}=\widetilde{F}_{j j}^{-1} U_{j}$. Defining $\mathrm{d}_{j}=\left[\begin{array}{ll}\Theta_{j} & G_{j}^{\prime}\end{array}\right]^{T}, R_{\theta_{j}}<$ $\mathrm{d}_{j}, \mathrm{~d}_{j}>$ is the covariance of $\Theta_{j}$ and is given by [6] 


\section{A Novel Hybrid Block INF Diagonalization (HBID) Precoding Technique for Capacity Improvement in MU-MIMO-OFDM System}

$$
Q_{\theta_{j}}=\left[\begin{array}{cc}
O_{K}+\gamma_{j}^{-1} & O_{K} \\
O_{K} & O-s Y
\end{array}\right]
$$

where. $\gamma_{j}=\widetilde{F}_{j j}^{-1} \widetilde{F}_{j j}$ Alluding to [14], L fulfills the condition in (12) in case and as it were in the event that $\mathrm{L}$ encompasses a least. In addition, the existing condition of the least shows that $Q_{\theta_{j}}$ has a J-spectral factorization, which could be attained by a closed-form canonical solution

$$
Q_{\theta_{j}}=J_{j} L J_{j}
$$

Where $\mathrm{J}=\operatorname{diag}\left\{O_{K},-O_{K}\right\}$, J ${ }_{j}$ is a $2 K \times 2 K$ invertible matrix. The minimum $\mathrm{L}$ can be defined as

$$
L_{\text {min }}=\left\|\breve{\mathrm{d}}_{\varphi_{j}}\right\|^{2}-\left\|\breve{\mathrm{d}}_{G^{\prime}{ }_{j}}\right\|^{2}
$$

Presumptuous $\sum_{j}=\left[\begin{array}{ll}\breve{\mathrm{d}}_{\varphi_{j}} & \breve{\mathrm{d}}_{E^{\prime}}{ }_{j}\end{array}\right]^{T}=J_{j}^{-1} \mathrm{~d}_{j}$, and

$$
J_{j}=\left[\begin{array}{ll}
J_{11} & J_{12} \\
J_{21} & J_{22}
\end{array}\right]
$$

Where, $J_{11}, J_{12}, J_{21}, J_{22}$ are the $k \times K$ matrices, correspondingly, we introduce a $k \times 1$ vector, $\psi$ satisfying $\|\psi\|_{\infty}=\max \left(\left|\psi_{1}\right|,\left|\psi_{2}\right|, \ldots,\left|\psi_{k}\right|\right)<1$ in order to guarnate $\mathrm{L}_{\text {min }}>0$ and we get

(16)

$$
\breve{\mathrm{d}}_{G^{\prime}{ }_{j}}=\psi \breve{\mathrm{d}}_{\varphi_{j}}
$$

Substituting (19) and $\Theta_{j}$ into $\Sigma_{j}$, we obtain

$$
\left[\begin{array}{c}
\breve{\mathrm{d}}_{\phi_{j}} \\
\psi \breve{\mathrm{d}}_{\varphi_{j}}
\end{array}\right]=J_{j}^{-1}\left[\begin{array}{c}
\emptyset_{j} \\
G_{j}^{\prime}
\end{array}\right]
$$

And

$$
\left[\begin{array}{ll}
J_{11} \breve{\mathrm{d}}_{\phi_{j}} & J_{12} \psi \breve{\mathrm{d}}_{\phi_{j}} \\
J_{21} \breve{\mathrm{d}}_{\phi_{j}} & J_{22} \psi \breve{\mathrm{d}}_{\phi_{j}}
\end{array}\right]
$$

(18) can be derived

$$
G_{j}^{\prime}=J_{2} J_{1}^{-1} \widetilde{F}_{j j}^{+} Z_{j}
$$

where $J_{1}=J_{11}+J_{12}$. Now, we find that the design of the $\mathrm{H}$-inf precoding primarily depends on J- spectral factorization of $Q_{\theta_{j}}$.

- Closed-Form Solution to the H-inf Precoding

Considering (15) and (16), we obtain $R_{\theta_{j}}=\left[\begin{array}{ll}\widehat{J}_{1,1} & \widehat{J}_{1,2} \\ \widehat{J}_{2,1} & \widehat{J}_{2,2}\end{array}\right]\left[\begin{array}{cc}O_{K} & 0 \\ 0 & -O_{K}\end{array}\right]\left[\begin{array}{ll}\widehat{J}_{1,1} & \widehat{J}_{1,2} \\ \widehat{J}_{2,1} & \widehat{J}_{2,2}\end{array}\right]^{F}$

Where $\widehat{J}_{1,1}, \widehat{J}_{1,2}, \widehat{J}_{2,1}, \widehat{J}_{2,2}$ is a group of conforming answers $\widehat{J}_{1,1}, \widehat{J}_{1,2}, \widehat{J}_{2,1}, \widehat{J}_{2,2}$ by Resolving (15).

\section{B. RF-PRECODER (HYBRID DIAGNO):}

Within the MU-MIMO frameworks, the generalized zero-forcing strategy (i.e., the conventional BD conspire) is infeasible to be for all intents and purposes actualized due to the tall taken a toll brought by the expansive number of RF chains as numerous as the radio antennas. By lessening the amount of RF chains $M_{B S}\left(M_{M S}\right)$ to far less than the antenna fundamentals $N_{B S}\left(N_{M S}\right)$ at both the BS and MSs, RF precoding matrix $\mathrm{F}$ is utilized at the $\mathrm{BS}$ and the RF combining matrix $Y_{k}$ is utilized at each MS to collect the expansive array gain given by the huge number of receiving antennas within the gigantic MU-MIMO channel. With the found $\mathrm{F}$ and all $W_{k}$ 's, the entire multi user equivalent baseband channel $F_{e q}$ k can be determined based on (4), which consists of all the equivalent channels for the MSs, namely $\tilde{F}_{k}, \mathrm{k}=1,2, \ldots, \mathrm{K}$. Finally, a low-dimensional BD processing, involving the design of $\mathrm{B}$ and all $T_{k}$ 's, can be performed at the baseband.

Where $\mathrm{B}=\operatorname{diag}\left\{B_{1}, B_{2}, \ldots, B_{k}\right\} \quad$ is a $K N_{S} \times K N_{S}$ diagonalmatrix. $\Sigma_{k}^{\left(N_{8}\right)} r$ epresents the first $N_{S} \times N_{S}$ block partition of $\Sigma_{k}$. As we choose DFT bases to construct $W_{k}$ 'sthe simplification step holds due to

$$
\begin{aligned}
& T_{k}^{H} Y_{k}^{H} Y_{k} T_{k}=\left(U_{k}^{\left(N_{S}\right)}\right)^{H} U_{k}^{\left(N_{S}\right)}=O_{N_{S}} . \\
& \mathrm{R}=\sum_{k=1}^{K} \log _{2}\left(\left|O_{N_{S}}+\frac{P B_{k}\left(T_{k}^{F} Y_{k}^{F} Y_{k} T_{k}\right)^{-1}\left(\Sigma_{k}^{\left(N_{8}\right)}\right)^{2}}{\sigma^{2} K N_{S}}\right|\right) \\
& \quad=\sum_{k=1}^{K} \log _{2}\left(\left|O_{N_{S}}+\frac{P B_{k}\left(\Sigma_{k}^{\left(N_{8}\right)}\right)^{2}}{\sigma^{2} K N_{S}}\right|\right)
\end{aligned}
$$

The outcome coming from the precoding method is given to CEM, which suppress the high amplitude by using $\eta=\frac{A}{\sqrt{\text { Paverage }}}$ equation, where $\mathrm{A}$ is the threshold value.

\section{CEM Technique for RF outcome:}

The following algorithm can be used to maintain better performance and the reduction of PAPR using CEM technique of MIMO-OFDM system.

Step 1: First initialize the CEM ratio and maximum number of repetition $\mathrm{M}$. Take $\mathrm{p}=1$.

Step 2: Generate the vector $\mathrm{Vb}$ in frequency response.

Step 3: Apply IFFT to $\mathrm{Vb}$ and get $\mathrm{Vb}=\mathrm{IFFT}(\mathrm{Vb})$.

Step 4: Compute the CEM ratio by the following equation

$$
\eta=\frac{A}{\sqrt{P_{\text {average }}}}
$$

where, $\eta$ is the CEM ratio

$\mathrm{A}$ is the threshold value

$\mathrm{P}_{\text {average }}$ is the average power of the signal before applying CEM technique.

Step 5: If the signal exceeds the threshold value compute the scaling factor $(\mu)$.

Step 6: Apply right circular shift to the scaling factor and it is represented as vs.

Step 7: PAPR reduction vector $v_{\mathrm{r}}=\mu \mathrm{v}_{\mathrm{s}}$

Step 8: Increment the value of $\mathrm{p}$ by 1 and repeat the steps 5-7 until all the peak above threshold A is suppressed.

\section{ACHIEVABLE SUM RATE AND PAPR PERFORMANCE}

\section{A. Achievable Sum Rate}

In this sub-section, the expression for achievable sum rate of MIMO-OFDM system is to be derived. To achieve sum rate three cases are taken and analyzed. The three cases are given below:

In case 1 , the device is completely synchronized without power amplifier and it is in non-linearity basis. The expression for case 1 with an $i^{\text {th }}$ component of $u^{\text {th }}$ user after the removal of cyclic prefix in the 
system is written as follows,

$$
\hat{x}_{u, i}=\frac{1}{N} \sum_{m=1}^{M} \sum_{n=0}^{N-1} E\left\{w_{m, u, n} H_{m, u, n}\right\} x_{u, i}+\xi_{u, i}
$$

where, $\xi_{u, i}$ is denoted as the effective noise component and it is given by,

$$
\xi_{u, i}=
$$

$\frac{1}{N} \sum_{m=1}^{M} \sum_{n=0}^{N-1} w_{m, u, n} H_{m, u, n} x_{u, i}$

$\frac{1}{N} \sum_{m=1}^{M} \sum_{n=0}^{N-1} E\left\{w_{m, u, n} H_{m, u, n}\right\} x_{u, i}+$

$\frac{1}{N} \sum_{q=1}^{N-1} \sum_{m=1}^{M} \sum_{n=0}^{N-1} w_{m, u, n} H_{m, u, n} x_{u, q} e^{\frac{j 2 \pi n(i-q)}{N}}+$

$\frac{1}{N} \sum_{\substack{k=1 \\ k \neq u}}^{K} \sum_{\substack{q=1 \\ q-1}}^{M-1} \sum_{m=1}^{M} \sum_{n=0}^{N-1} w_{m, u, n} H_{m, u, n} x_{u, q} e^{\frac{j 2 \pi n(i-q)}{N}}+$

$\eta_{\mathrm{u}, \mathrm{i}}$

In case 2 , the system is affected due to the presence of instability in frequencies which is called CFO by $\mathrm{u}^{\text {th }}$ user $\mathrm{Eu}$ which is represented by the following equation. The equation is expressed fora received signal having ith component of uth user is given below.

$$
\hat{x}_{u, i}=
$$

$\frac{1}{N} \sum_{m=1}^{M} \sum_{n=0}^{N-1} \sum_{k=1}^{K} \sum_{q=0}^{N-1} w_{m, k, n} H_{m, u, n} x_{k, q} e^{\frac{j 2 \pi n(i-q)}{N}} e^{\frac{j 2 \pi E_{u} i}{N}}+$

$\eta_{u, i}$

In case 3 , the device is disturbed by the power amplifier with non-linearity and the expression is given for the SC-FDP MIMO system having $i^{\text {th }}$ sample for input signal for power amplifier of $\mathrm{m}^{\text {th }}$ antenna.

$$
y_{m, i}=\frac{1}{N} \sum_{k=1}^{K} \sum_{n=0}^{N-1} \sum_{q=0}^{N-1} w_{m, k, n} x_{k, q} e^{\frac{j 2 \pi n(i-q)}{N}}
$$

\section{B. Peak-to-Average Power Ratio (PAPR)}

If the signal's constellations are identical, the PAPR of OFDM signal without precoder is greater than the PAPR of signal with precoders. This high PAPR in the OFDM system leads to in-band distortion and out of band emission which can be mitigated by RF amplifiers. This creates a major problem in MIMO systems if the number of RF amplifiers is directly proportional to $\mathrm{M}$. The $\mathrm{m}^{\text {th }}$ antenna present at the base station produces a transmitted signal having the PAPR value which has been derived from the following equation.

$$
P A P R_{m}=\frac{\max \left|y_{m, i}\right|^{2}}{\frac{1}{N} \sum_{i=0}^{N-1}\left|y_{m, i}\right|^{2}}
$$

The distribution of $y_{m, i}$ can be approximated by Gaussian. CCDF of PAPR for OFDM system can be expressed by, $P_{r}\left(P A P R_{m}>\gamma_{0}\right)=1-\left(1-e^{-\gamma_{0}}\right)^{2.8 N}$

\section{5.3 BER in OFDM system:}

BER is an important parameter which is used to determine the output signal performance by comparing signal with how much bits are received by error and how mush bits are transmitted over a time interval. It is the ratio of received error bits by total bits transmitted over an interval. It does not have any unit; this is only measured as percentage.

$B E R=$ No. of bit errors recieved / Total number of bits transmitted

In modern mobile communication, BER takes an important role, because it is disturbed the received signal when the signal is transmitted over a long distance. It may be occur because of fading, noise, interference, unwanted sound, timing problem, low signal strength, weak channel, attenuation, channel noise. This can only improve by transmitting high strength signal rather than low strength signal or by applying slow modulation, demodulation and line coding.

\section{SNR in OFDM system:}

SNR is a measurement tool which compares the amount of transmitted signal strength to noise appears on the signal. It can be well-defined as signal power divided by noise power. The unit of SNR is DB which stands for decibel. It is the number which measure the two signals quantity such as amplitude. It is really a logarithmic propulsion whose primary reason is to scale an expansive estimation range down to a much slighter and more useable range. When the SNR is high, that means more signal and less noise. It is good for communication. In our proposed method the SNR performance is improved compared with previous precoding methods.

\section{SIMULATION RESULT:}

The MU-MIMO system is simulated in MATLAB as shown in Fig [5-14]. The modulation technique used in MIMO- OFDM system is Quadrature Amplitude (QAM) with 128 subcarriers. In this system, QAM is preferred because it reduces the bit error rate and increases the data rate when compared to Binary Phase Shift Keying (BPSK).

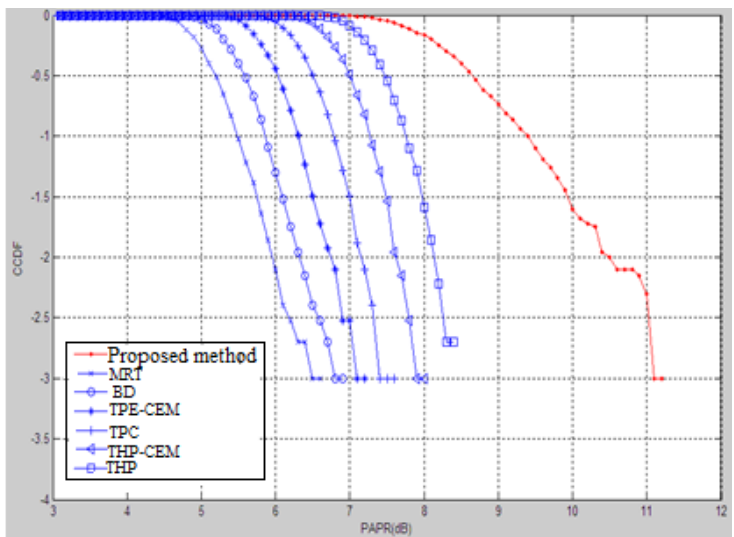

Fig.3. PAPR vs CCDF for 16-QAM, 128 sub-carriers, $\mathrm{U}=\mathbf{3 0}$

Fig. 3 shows the simulation result for PAPR vs CCDF of various precoding technique in OFDM systems for 16-QAM. The performance of the PAPR value for various precoding such as THP, THP-CEM, TPE, TPE-CEM, BD, MRT techniques are compared with proposed method. The plots are clearly shown in Fig. 3. When the signal is transmitted through OFDM system the PAPR is high. But with the help of CEM and Precoding techniques, the PPAR value can be reduced. 


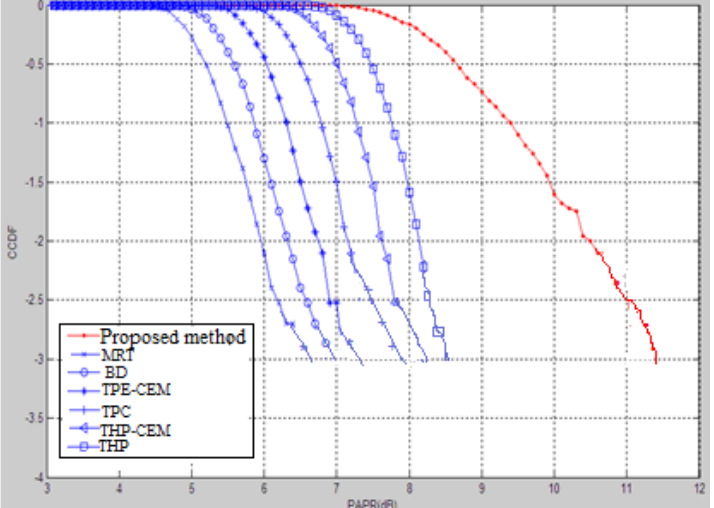

Fig.4. PAPR vs CCDF for 64-QAM, 128 sub-carriers, $\mathrm{U}=\mathbf{3 0}$

The simulation result of PAPR vs CCDF for various precoding techniques for 64-QAM, 128-sub carriers and $\mathrm{U}=30$ is shown in Fig. 6. The proposed system high performance when compared with previous OFDM system because Proposed method reduces the PAPR value for a considerable rate which is clearly shown in the above Fig. 4.

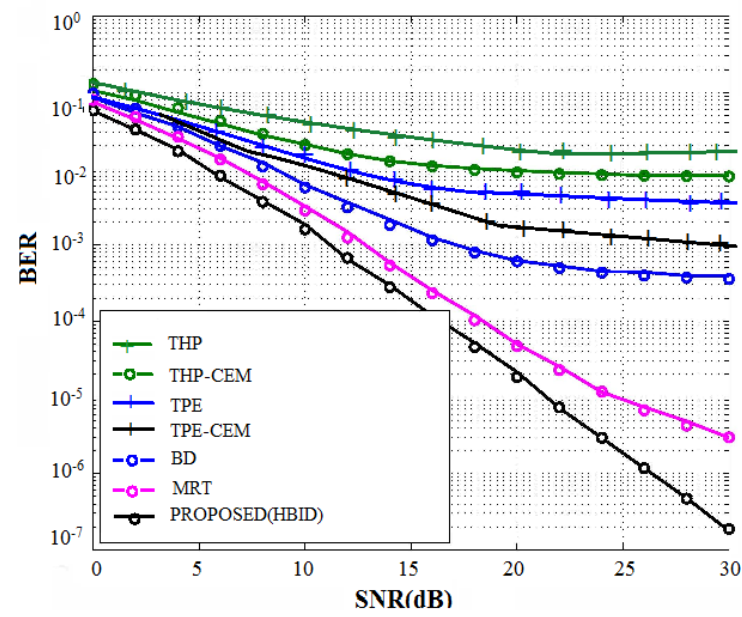

Fig.5. SNR vs BER for 16-QAM, 128 sub-carriers, $\mathbf{U}=\mathbf{3 0}$

Here, analyze the performance of the proposed work using 16QAM and 128 subcarriers. In the proposed method uses the HBID technique and the BER performance is compared with various previous techniques. The goal of the projected method is to a reduction of PAPR in the Orthogonal FDM scheme.

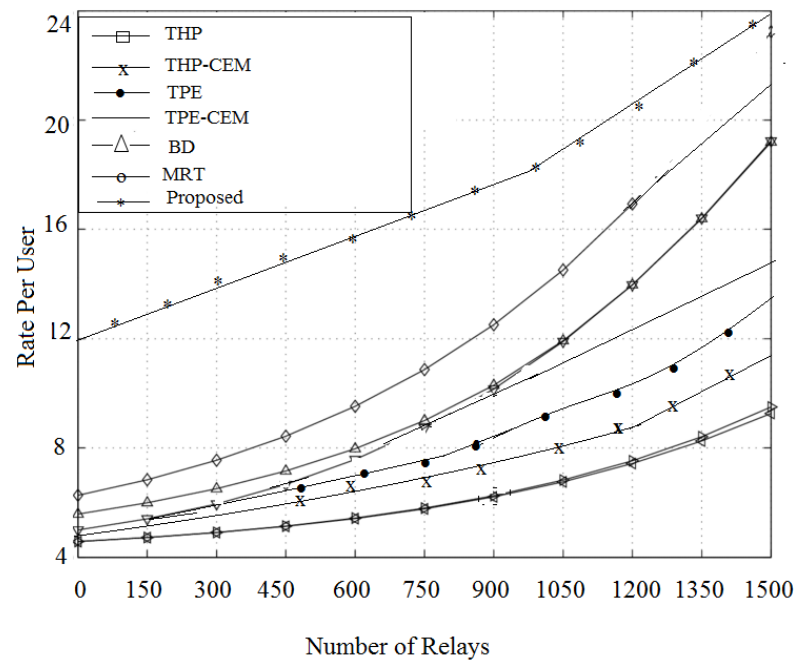

Fig.6. Number of Relay vs Rate per user for, 16-QAM, 128 Subcarriers, $U=30$
The simulation result for the number of relays and rate per users for 16-QAM, 128 subcarriers, $\mathrm{U}=30$ with proposed technique is depicted in Fig.6. For obtaining better performance the number of users must be high. This is because at high SNR the achievable sum rate mitigates the inter cell interference and intra cell interference based on the number of users. Compared to the plot the proposed technique improves the rate per user by $75 \%$ when compare with THP method.

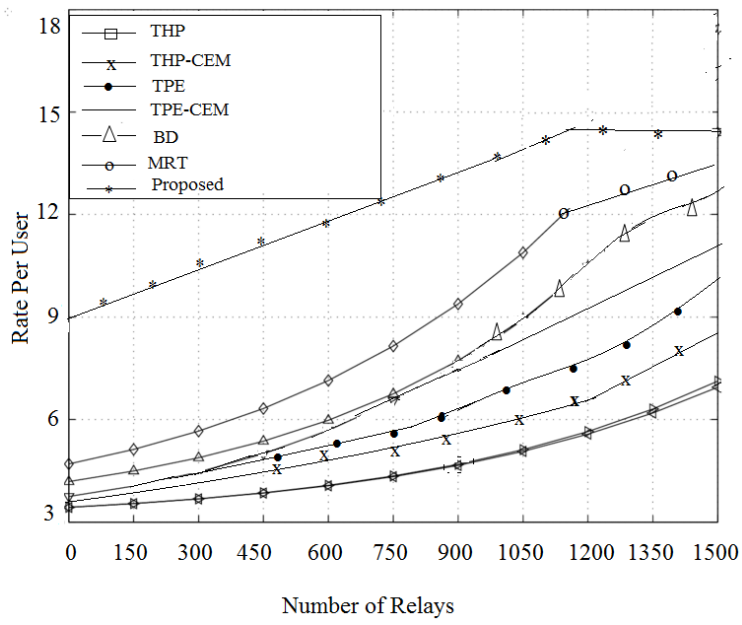

Fig.7. Number of Relay vs Rate per user for, 64-QAM, 128 Subcarriers, $U=30$

Fig.7. shows the simulation result for the number of relays and rate per users for 64-QAM, 128 subcarriers, $\mathrm{U}=30$. Based on the threshold value 1.4 used in CEM technique the user rate can be increased. The threshold value can be maintained as 1.4 for obtaining best performance. This optimized threshold value could be found from trial and error method. This Fig. 13 clearly shows when compared to THP, there is $46 \%$ increase in the user rate.

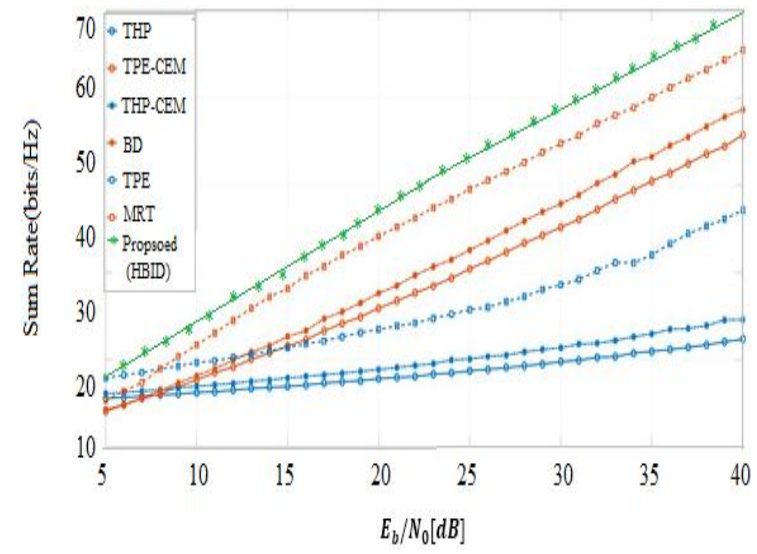

Fig.8. Eb/N0 vs Sum Rate for, 16-QAM, 128 Subcarriers and $\mathbf{U}=\mathbf{3 0}$

Fig.8. shows that the comparison various precoding technique with proposed method. When the Eb/N0 value is increased the sumrate is also increases. But the proposed method improves the sum rate $50 \%$ when compared with THP method. This clearly shows that the precoding techniques has the best performance when compared to the other CEM methods. 


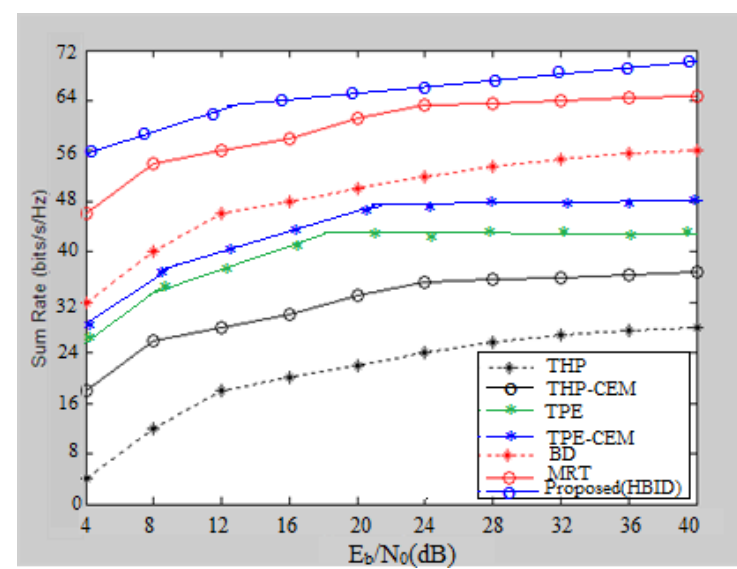

Fig.9. Eb/N0 vs Sum Rate for, 64-QAM, 128 Subcarriers and $\mathrm{U}=\mathbf{3 0}$

The Fig.9. shows Eb/N0 vs sum rate comparison between the previous precoding technique with proposed technique for 64-QAM, 128 subcarriers and $U=30$ is shown in Fig. 5 and that is clearly shows that the performance of rate per users increases with proposed technique.

\section{CONCLUSION}

The proposed HBID precoding technique is used in MU-MIMO-OFDM to establish a low complexity MIMO system and the PAPR value is reduced with the help of CEM technique by cutting down the amplitude of the given signal. So, the PAPR value of the proposed system is reduced and the system performance is increased when compared to the existing system. Also, the various types of precoding techniques such as THP, TPE, BD, MRT with CEM and without CEM are discussed and compared with proposed method. Based on the simulation results the HBID has better performance because it reduces the PAPR and number of relays. At the same time, the proposed system increases the sum rate and rate per users. When the HBID technique is compared to the THP without CEM the sum rate is increased as $57 \%$ as well as the user rate is increased as $49 \%$.

\section{REFERENCES}

1. Marius-Constantin O.S. Popescu and Nikos E. Mastorakis, "The use of MIMO technologies in wireless communication networks", 3rd International Conference on Communication and Information Technology, pp. 139-145.

2. J. Mietzner, R. Schober, L. Lampe, W. H. Gerstacker, and P. A. Hoeher, "Multiple-antenna techniques for wireless communications-A comprehensive literature survey," IEEE Commun. Surv. Tuts., vol. 11, no. 2, pp. 87-105, Jun. 2009.

3. S. Sugiura, S. Chen, and L. Hanzo, "A universal space-time architecture formultiple-antenna aided systems," IEEE Commun. Surv. Tuts., vol. 14, no. 2, pp. 401-420, Jan. 2012.

4. A. Chockalingam and B. S. Rajan, Large MIMO Systems. Cambridge,U.K.: Cambridge Univ. Press, 2014.

5. Thomas L Marzetta, "Non cooperative Cellular Wireless with Unlimited Numbers of Base Station Antennas" IEEE Trans. Comm., vol. 9, no. 11, pp.3590-3600, November 2010.

6. Heshani Gamage, NandanaRajatheva and Matti Latva-aho, "Performance and PAPR Analysis of Single-Carrier Massive MIMO Systems with Channel Imperfections", Vehicular Technology Conference (VTC-Fall), 2017.

7. F. Boccardi, R. W. Heath, A. Lozano, T. L. Marzetta, and P. Popovski, "Five disruptive technology directions for 5G," IEEE Commun. Mag., vol. 52, no. 2, pp. 74-80, Feb. 2014.

8. E. G. Larsson, O. Edfors, F. Tufvesson, and T. L. Marzetta, "Massive MIMO for next-generation wireless systems," IEEE Commun. Mag., vol. 52, no. 2, pp. 186-195, Feb. 2014.
9. Ping Yang, Yue Xiao and Yong Liang Guan, "Single-Carrier SM-MIMO: A Promising Design for Broadband Large-Scale Antenna Systems", IEEE Communications Surveys \& Tutorials, vol. 18, no. 3, pp. 1687-1716, Feb. 2016.

10. Sven Jacobsson, Giuseppe Durisi, Mikael Coldrey and Christoph Studer, "Massive MU-MIMO-OFDM Downlink with One-Bit DACs and Linear Precoding", IEEE Global Communications Conference, Dec. 2017.

11. Stefano Buzzi, Carmen D'Andrea, Tommaso Foggi, Alessandro Ugolini and Giulio Colavolpe, "Single-Carrier Modulation versus OFDM for Millimeter-Wave Wireless MIMO", in IEEE Transaction on Communications, vol.66, no. 3, pp. 1335-1348, Nov. 2017

12. Sajjad Ali, Zhe Chen and Fuliang Yin, "Eradication of pilot contamination and zero-forcing precoding in the multi-cell TDD massive MIMO systems", IET Communications, vol.11, no. 13, pp. 2017-2034, Oct. 2017.

13. Jubin Jose, Alexei Ashikhmin, Thomas L. Marzetta and Sriram Vishwanath, "Pilot Contamination and Precoding in Multi-Cell TDD Systems", IEEE Transactions on Wireless Communications, vol. 10, no. 8, Aug. 2011.

14. AntoniosPitarokoilis, Saif Khan Mohammed and Erik G. Larsson, "On the Optimality of Single-Carrier Transmission in Large-Scale Antenna Systems", IEEE Wireless Communications Letters, vo. 1, no. 4, Aug. 2012.

15. Mohammed Ali Mohammed MOQBEL, Wangdong,and Al-marhabi Zaid Ali, "MIMO Channel Estimation Using the LS and MMSE Algorithm", IOSR Journal of Electronics and Communication Engineering (IOSR-JECE), vol. 12, no. 1, pp. 13-22, Feb. 2017.

16. J.P. Coon and M. A. Beach, "An Investigation of MIMO Single-Carrier Frequency-Domain MMSE Equalization”, London Communications Symposium, pp. 237-240, 2002.

17. Weiheng Ni, and Xiaodai Dong, "Hybrid Block Diagonalization for Massive Multiuser MIMO Systems", IEEE TRANSACTIONS ON COMMUNICATIONS, vol. 64, no. 1, pp:201-211, Jan 2016.

18. [18] Peng Xu, Jiang Zhou Wang and Jinkuan Wang, "Multi-cell H-inf Precoding in Massive MIMO Systems", IEEE ICC 2014 - Signal Processing for Communications Symposium, pp:4472-4476. 